\title{
Akhmediev Breathers and Peregrine Solitary Waves in a Quadratic Medium
}

\author{
Fabio Baronio 1 ,* \\ ${ }^{1}$ INO CNR and Dipartimento di Ingegneria dell'Informazione, \\ Università di Brescia, Via Branze 38, 25123 Brescia, Italy
}

\begin{abstract}
We investigate the formation of optical localized nonlinear structures, evolving upon a non-zero background plane wave, in a dispersive quadratic medium. We show the existence of quadratic Akhmediev breathers and Peregrine solitary waves, in the regime of cascading second-harmonic generation. This finding opens a novel path for the excitation of extreme rogue waves and for the description of modulation instability in quadratic nonlinear optics.
\end{abstract}

In dispersive optical media, with cubic Kerr nonlinearity, the nonlinear Schrödinger equation (NLSE) provides a central description of a variety of nonlinear localization effects [1. In fact, since the 1970s there has been wide and continued investigation into the properties of analytic soliton solutions of the NLSE [2, both for their intrinsic scientific interest, as well as for their potential to provide new insights into important applications such as optical propagation in nonlinear waveguides 3. For the case of a self-focussing nonlinearity, the most celebrated solution of this type is very likely the propagation-invariant hyperbolic secant bright soliton, but there is also an extensive literature studying various types of solitons on finite background consisting of a localized nonlinear structure evolving upon a nonzero background plane wave [4 6].

Solitons on finite background have recently attracted significant interest as their localization dynamics have been proposed as an important mechanism underlying the formation of the infamous extreme amplitude rogue waves on the surface of the ocean [7, 8]. Much of this work has also been motivated by a parallel research effort using nonlinear optical fibre systems to implement controlled experiments studying NLSE dynamics and rogue waves in an optical context 9 15]. Many of the recent studies have focussed on the characteristics of the Akhmediev breather [6], a soliton on finite background solution which is excited from a weak periodic modulation and which is localized in the longitudinal dimension as it undergoes growth and decay. Experiments in optics have demonstrated important links to modulation instability (MI) [9, 12 14]: of importance has been the realization that many properties of MI previously described only approximately (via numerical or truncated mode approaches) can in fact be described almost exactly using Akhmedievbreathers. Another significant application of the theory of Akhmediev breathers has been to design experiments generating the rational Peregrine soliton [5], an important and limiting case of a solitons on finite background solution that is localised in both transverse and longitudinal dimensions [10, 11].

In dispersive optical media, with quadratic nonlinearity, the existence of localized nonlinear structures evolving upon a non-zero background plane wave remained unexplored to date.

In this Letter, we investigate the formation of lo- calized nonlinear structures, evolving upon a non-zero background plane wave, in optical systems described by second harmonic generation (SHG) equations [16. We show the existence of quadratic Akhmediev breathers and Peregrine solitary waves, in the regime of cascading SHG [17. To our knowledge, no studies have explicitly characterized nonlinear breather localization in any systems described by the SHG. This finding opens a novel path for the excitation of extreme rogue waves, and for the description of MI [18] in quadratic media.

In transparent dispersive media, with quadratic nonlinearity, the interaction of the fundamental frequency $(\mathrm{FF})$ and the second harmonic $(\mathrm{SH})$ envelopes $u_{1,2}=$ $u_{1,2}(\tau, \xi)$, at frequency $\omega_{0}$ and $2 \omega_{0}$ respectively, obeys coupled equations that, in dimensionless form, read as 17, 18

$$
\begin{gathered}
i u_{1 \xi}-\frac{\beta_{1}}{2} u_{1 \tau \tau}+u_{2} u_{1}^{*} e^{-i \delta k \xi}=0, \\
i u_{2 \xi}+i v u_{2 \tau}-\frac{\beta_{2}}{2} u_{2 \tau \tau}+u_{1}^{2} e^{i \delta k \xi}=0,
\end{gathered}
$$

where $\xi \equiv z / z_{d} \equiv z\left|\beta_{1}\right| / t_{0}^{2}$ is the propagation distance in units of the dispersion lenght $z_{d}=t_{0}^{2} /\left|\beta_{1}\right|$ associated with the FF dispersion $\beta_{1}^{\prime \prime}=d^{2} k /\left.d \omega^{2}\right|_{\omega_{0}} ; \tau=\left(t-z / v_{1}\right) / t_{0}$ is the time in a reference frame traveling with the FF group velocity $v_{1}^{-1}=d k /\left.d \omega\right|_{\omega_{0}}$ ( $t_{0}$ being an arbitrary time scale); $\beta_{1}=\operatorname{sign}\left(\beta_{1}^{\prime \prime}\right), \beta_{2}=-\operatorname{sign}\left(\beta_{2}^{\prime \prime}\right)\left|\beta_{2}^{\prime \prime} / \beta_{1}^{\prime \prime}\right|$, with $\beta_{2}^{\prime \prime}=d^{2} k /\left.d \omega^{2}\right|_{2 \omega_{0}} ; \delta k=\Delta k z_{d}$ is the normalized wave-vector mismatch, with $\Delta k=k_{2}-2 k_{1}, k_{1}=\left.k\right|_{\omega_{0}}$, $k_{2}=\left.k\right|_{2 \omega_{0}} ; v \equiv z_{d} / z_{w}$ is the ratio between the dispersion and the walk-off length $z_{w} \equiv t_{0} /\left(v_{2}^{-1}-v_{1}^{-1}\right)$, where $v_{2}^{-1}=d k /\left.d \omega\right|_{2 \omega_{0}}$ is the $\mathrm{SH}$ group velocity. The fields $u_{1}=\chi z_{d} A_{1}$ and $u_{2}=\chi z_{d} A_{2}$, where $|A|_{1,2}^{2}$ measure directly the intensity (in watts per square meter) if we set the nonlinear coefficient $\chi=\omega_{0}\left[2 /\left(c^{3} \epsilon_{0} n_{\omega 0}^{2} n_{2 \omega 0}\right)\right]^{1 / 2} d^{(2)}$ and $d^{(2)}$ is the effective susceptibility element (in meter per volt).

We point out that Eqs. (11) also govern the interaction of beams focused in one transverse dimension, making the substitution $t \rightarrow x, \beta_{i}^{\prime \prime} \rightarrow 1 / 2 k_{i}$, and being $v$ the birefingence walk-off.

In the cascading regime, at large phase-mismatch, by using the $\mathrm{SH}$ asymptotic expansion and the method of repeated substitution, we arrive at a single FF evolution equation, at first order approximation [17]:

$$
i \rho_{\xi}-\frac{\beta_{1}}{2} \rho_{\tau \tau}+\sigma|\rho|^{2} \rho=0,
$$


where, for sake of clarity, we have defined $u_{1}=\rho, u_{2} \simeq$ $\rho^{2} e^{i \delta k \xi} / \delta k, \sigma=1 / \delta k$. We emphasize that the validity of the mapping of the FF wave evolution of Eqs. (1) into Eq. (2) entails weak overall FF to $\mathrm{SH}$ conversion.

Over the last decades, the cascading regime has been successfully exploited in the demonstration of quadratic spatial solitary waves [19, soliton bouncing at nonlinear interfaces [20, temporal solitary waves [21, steepening and spectral dynamics [22, 23, temporal compression [24, 25], and shock waves [26].

In this Letter, we focus our attention on i) anomalous dispersion $\left(\beta_{1}=-1\right)$ and effective self-focusing nonlinearities $(\sigma>0)$, ii) normal dispersion $\left(\beta_{1}=1\right)$ and effective self-defocusing nonlinearities $(\sigma<0)$, which both yield the integrable focusing NLSE.

We proceeded to demonstrate the existence of $\mathrm{FF}$ Akhmediev breathers of the SHG Eqs. (1), which are predicted theoretically through the mapping with the NLSE Eq. (2). We consider the standard case in the experiments such that only a FF pulse is launched in the quadratic medium.

A form of the Akhmediev breathers soliton solution of the NLSE Eq. (2), under the condition $\beta_{1} \sigma<0$, can be expressed as [6, 11]:

$\rho=\rho_{0}\left[\frac{(1-4 a) \cosh \left(b \xi_{n}\right)+\sqrt{2 a} \cos \left(\Omega \tau_{n}\right)+i b \sinh \left(b \xi_{n}\right)}{\sqrt{2 a} \cos \left(\Omega \tau_{n}\right)-\cos \left(b \xi_{n}\right)}\right]$,

where $\rho_{0}=\eta e^{i \xi_{n}}, \tau_{n}=\sqrt{\left|\sigma / \beta_{1}\right| \eta^{2}} \tau, \xi_{n}=|\sigma| \eta^{2} \xi ; \eta$ is the amplitude background, $\Omega$ is the modulation frequency, $a=1 / 2\left(1-\Omega^{2} / 4\right)$, where $0<a<1 / 2$ determines the frequency that experience gain, and $b=[8 a(1-2 a)]^{1 / 2}$ determines the instability growth.

We verified numerically the theorethical prediction of the FF Akhmediev breather dynamics $u_{1}=\rho$ of the Eqs. (1), where $\rho$ is the NLSE Akhmediev breather soliton solution (3), with only the FF wave at the input of the quadratic medium. Thus, in our numerics, the input solitary wave envelopes $u_{1,2}$ at $\xi=\xi_{0}$ are given by the expression $u_{1}\left(\tau, \xi_{0}\right)=\rho\left(\tau, \xi_{0}\right), u_{2}\left(\tau, \xi_{0}\right)=0$.

Figure 1(a), (b) report the spatiotemporal numerical envelopes evolution $\left|u_{1}\right|$ at $\mathrm{FF}$, and $\left|u_{2}\right|$ at $\mathrm{SH}$ of a typical quadratic cascading Akhmediev breather solitary wave, in the $(\tau, \xi)$ plane. The numerical evolution of the $\mathrm{FF}$ Akhmediev breather in Fig. 1(a) maps very well on the evolution predicted by the soliton solution (3) of the NLSE Eq. (2). The inset in Fig. 11(a) reports the comparison between the numerical (blue line) and the analytical (dot red line) envelope profile of the Akhmediev breather at his maximal amplitude, at $\xi=0$. In this particular case, we estimate the relative error between the numerical FF wave of the Eqs. (1) and the analytical formula (3) to be around $4 \%$, because of the FF depletion due to $\mathrm{SH}$ wave generation.

Very interestingly, the generated SH wave in Fig. 1)(b), apart from low amplitude dispersive waves, is of breather nature and strictly locked with the FF component, indicating the existence of genuine quadratic Akhmediev
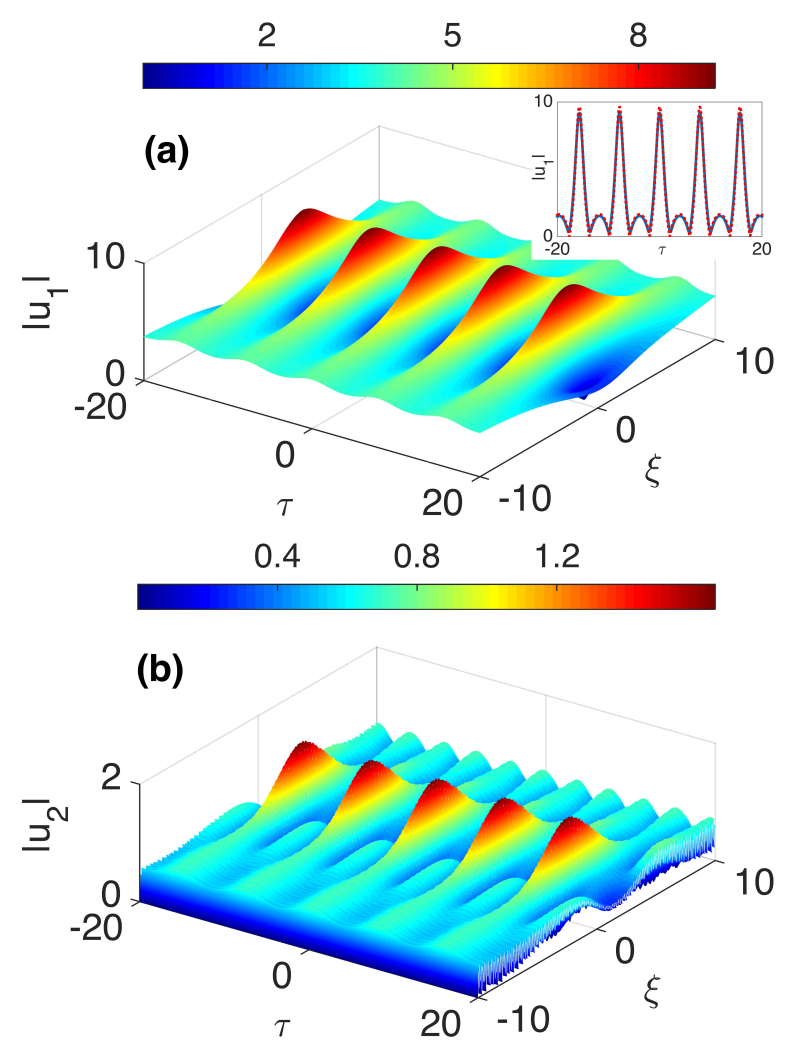

FIG. 1. (a), (b) Spatiotemporal numerical envelopes evolution, $\left|u_{1}\right|$ at $\mathrm{FF}$ and $\left|u_{2}\right|$ at $\mathrm{SH}$, of a typical quadratic cascading Akhmediev breather solitary wave, in the $(\tau, \xi)$ plane. The inset reports the comparison between the numerical (blue line) and the analytical (dot red line) envelope profile of the Akhmediev breather, at $\xi=0$. Here $\beta_{1}=-1, \beta_{2}=-1, v=$ $0, \delta k=50 ; \eta=4, \Omega=\sqrt{2}(a=1 / 4, b=1)$.

solitary breathers.

Next, we proceeded to demonstrate the existence of Peregrine solitary waves of the SHG Eqs. (1), again predicted theoretically through the mapping with the NLSE Eq. (2).

A rational form of the Peregrine soliton solutions of the Eq. 22), under the condition $\beta_{1} \sigma<0$, can be expressed as [5, 10:

$$
\rho(\tau, \xi)=\rho_{0}\left[1+\frac{4\left(1+2 i \xi_{n}\right)}{1+4 \tau_{n}^{2}+4 \xi_{n}^{2}}\right]
$$

where $\rho_{0}=\eta e^{i \xi_{n}}, \tau_{n}=\sqrt{\left|\sigma / \beta_{1}\right| \eta^{2}} \tau, \xi_{n}=|\sigma| \eta^{2} \xi, \eta$ is the amplitude background. The amplitude $|\rho(\tau, \xi)|$ is peaked at $\xi=0$ with the maximum value $3|\eta|$ at $\tau=0$. Note that the rational solution (4) can be derived from (3), in the asymptotic case $a \rightarrow 1 / 2$.

In the case at hand, we assumed that the FF wave propagates tightly locked with the $\mathrm{SH}$ wave, and we tested the theoretical prediction of a genuine quadratic Peregrine solitary dynamics $u_{1}=\rho$ at the $\mathrm{FF}$, and $u_{2}=$ $\rho^{2} e^{i \delta k \xi} / \delta k$ at the SH, where $\rho$ is the NLSE Peregrine soliton solution (4). Thus, in our numerics, the input solitary 

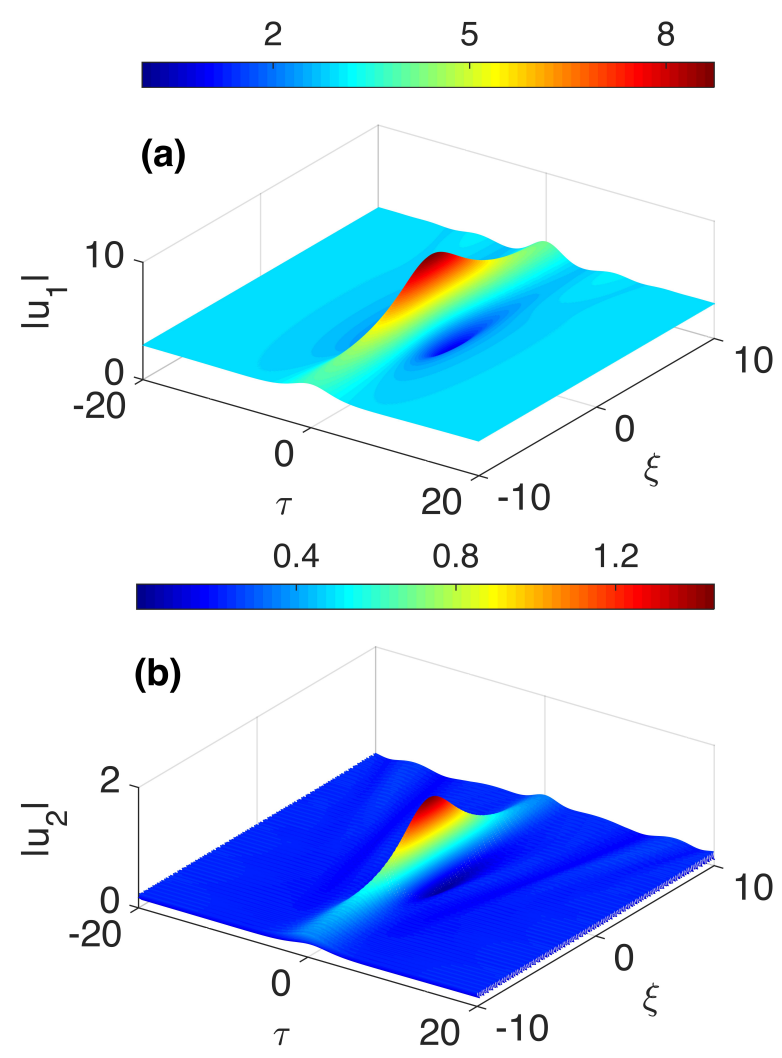

FIG. 2. (a), (b) Spatiotemporal numerical envelopes evolution, $\left|u_{1}\right|$ at $\mathrm{FF}$ and $\left|u_{2}\right|$ at $\mathrm{SH}$, of a typical quadratic cascading Peregrine solitary wave, in the $(\tau, \xi)$ plane. Here $\beta_{1}=1, \beta_{2}=1, v=0, \delta k=-50$ and $\eta=2.5$.

wave envelopes $u_{1,2}$ at $\xi=\xi_{0}$ are given by the expression $u_{1}\left(\tau, \xi_{0}\right)=\rho\left(\tau, \xi_{0}\right), u_{2}\left(\tau, \xi_{0}\right)=\rho^{2}\left(\tau, \xi_{0}\right) e^{i \delta k \xi_{0}} / \delta k$.

Figure 2(a), (b) report the spatiotemporal numerical envelopes evolution $\left|u_{1}\right|$ at FF, and $\left|u_{2}\right|$ at $\mathrm{SH}$ of a typical quadratic cascading Peregrine solitary wave, in the $(\tau, \xi)$ plane. The numerical evolution of the quadratic Peregrine components at the FF and $\mathrm{SH}$ in Fig. 2 is very well predicted by the theoretical model. We estimate the error between the numerical $\mathrm{FF}$ and $\mathrm{SH}$ wave of the Eqs. (1) and the analytical predictions to be lower than $1 \%$.

MI in dispersive media with quadratic nonlinearity involves the spontaneous generation of sideband pairs around both the $\mathrm{FF}$ and $\mathrm{SH}$ frequencies [18. MI may occur in the anomalous (i.e, $\beta_{1,2}<0$ ) and in the normal (i.e, $\beta_{1,2}>0$ ) dispersion regimes; $\mathrm{MI}$ occurs also if the $\mathrm{FF}$ and the $\mathrm{SH}$ pulses are in different regimes of dispersion (i.e., $\left.\beta_{1} \beta_{2}<0\right)$. In the initial evolution of MI, the spectral sidebands associated with the instability experience exponential amplification at the expense of the pumps, but the subsequent dynamics are more complex and display cyclic energy exchange between spectral modes [27, 28]. In particular, MI seeded from noise results in a series of high-contrast peaks of different intensity.

Figure $3(a)$, (b) plot a density map of the numerical
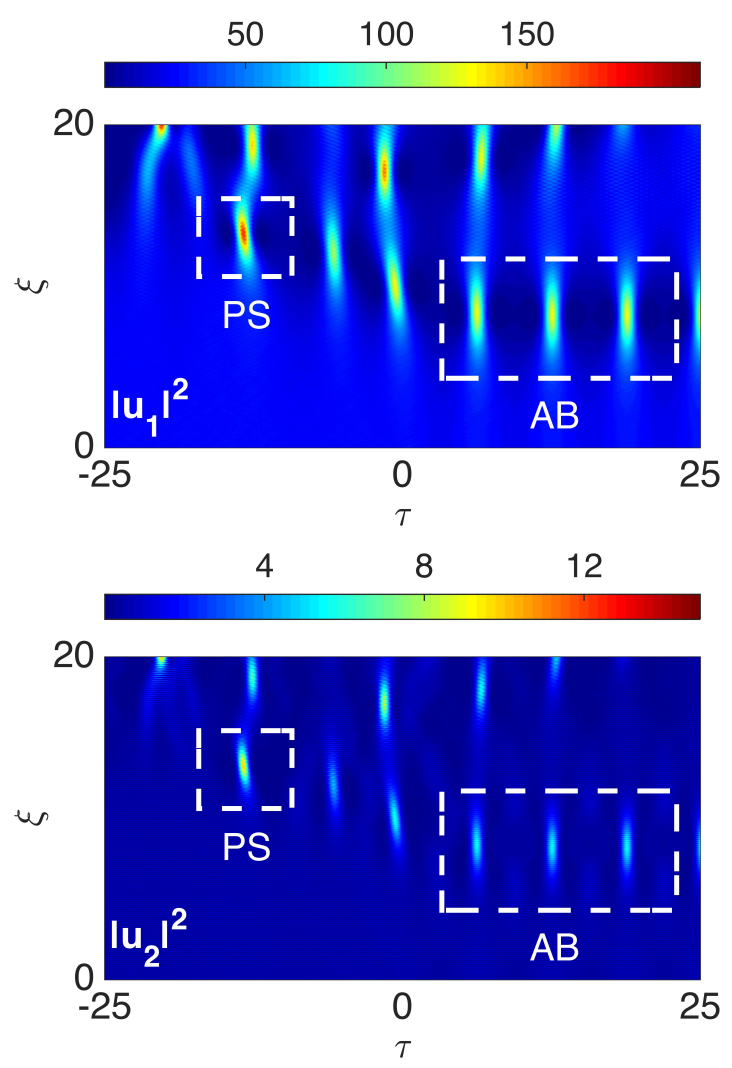

FIG. 3. Density map of the numerical temporal evolutions of FF (a) and SH (b) waves, in the $(\tau, \xi)$ plane. Here $\beta_{1}=-1$, $\beta_{2}=-1, v=0, \delta k=-50$. At the input, $u_{1}(\tau, 0)=u_{10}+w$, with $u_{10}=5, w$ one-photon-per-mode noise, $u_{2}(\tau, 0)=0$. The label PS indicates Peregrine solitary structures, the label $\mathrm{AB}$ indicates Akhmediev breathers.

temporal evolutions of $\mathrm{FF}$ and $\mathrm{SH}$ waves, respectively, of a FF chaotic field triggered by one-photon-per-mode noise superimposed on a continuous-wave background at the input of the quadratic medium. In our numerics, the input wave envelopes $u_{1,2}$ at $\xi_{0}$ are given by the expression $u_{1}\left(\tau, \xi_{0}\right)=u_{10}+w, u_{2}\left(\tau, \xi_{0}\right)=0$, where $u_{10}$ represent the continuous wave amplitude, $w$ the noise. Figure 3(a), (b) report the emergence of an irregular series of temporal peaks, triggered by MI, around $\xi=5$. Examining particular features of the evolution map (i.e., the periodicity in $\tau, \tau-\xi$ localizations, the peak intensity values) reveals clear signatures of quadratic Akhmediev solitary breathers and Peregrine solitary waves. Of course, observing ideal breathers or Peregrine structures is not expected given the random initial conditions, but it is remarkable how the quadratic breathers and Peregrine solitary solutions can be mapped closely to the noise-generated structures. These results confirm that quadratic Akhmediev breathers and Peregrine waves can provide insights into structures emerging from noiseseeded MI in SHG precesses.

Let us briefly discuss possible experimental settings in 
nonlinear optics for the observation of quadratic extreme solitary wave dynamics. As to the temporal pulse dynamics, one may consider optical temporal propagation in a bulk $\mathrm{BBO}, \mathrm{KTP}$ or $\mathrm{LiNbO}_{3}$ crystal in the regime of high phase mismatch, where quadratic solitary waves, temporal compression, spectral shift controls and steepening effects have been demonstrated (e.g., see the experimental setups of Refs. [21 24]). As to spatial dynamics, one may consider the spatial propagation in a bulk or in a planar quadratic $\mathrm{LiNbO}_{3}$ crystal, where spatial quadratic solitary waves, bouncing at nonlinear interfaces, and solitary interactions have already been demonstrated (e.g., see the experimental setups of Refs. [19, 20]).
In conclusion, we investigated the formation of optical localized nonlinear structures, evolving upon a non-zero background plane wave, in dispersive quadratic media. We have demonstrated analytically and numerically the existence of quadratic Akhmediev breathers and Peregrine solitary waves, in the regime of cascading secondharmonic generation. On one hand, this finding opens a novel path for the prediction, excitation and control of extreme rogue waves in quadratic media; on the other hand, this result provides novel insights in MI dynamics.

Funding The present research was partially supported by the Italian Ministry of University and Research (MIUR) (2012BFNWZ2).
* fabio.baronio@unibs.it

[1] N. Akhmediev and A. Ankiewicz, Solitons, Nonlinear Pulses and Beams (Chapman and Hall, 1997).

[2] V. E. Zakharov and A.B. Shabat, Zh. Eksp. Teor. Fiz. 61, 118-134 (1971).

[3] G.P. Agrawal, Nonlinear Fiber Optics (Academic Press, 2007).

[4] Y. C. Ma, Stud. Appl. Math. 60, 43-58 (1979).

[5] D. H. Peregrine, J. Austral. Math. Soc. Ser. B 25, 16-43 (1983).

[6] N. Akhmediev and V.I. Korneev, Theor. Math. Phys. 69, 1089-1093 (1986).

[7] C. Kharif, E. Pelinovsky, and A. Slunyaev, Rogue Waves in the Ocean (Springer, 2009).

[8] M. Onorato, S. Residori, and F. Baronio, Rogue and Shock Waves in Nonlinear Dispersive Media (Springer, 2016).

[9] J.M. Dudley, G. Genty, F. Dias, B. Kibler, and N. Akhmediev, Opt. Express 17, 21497-21508 (2009).

[10] B. Kibler, J. Fatome, C. Finot, G. Millot, F. Dias, G. Genty, N. Akhmediev, and J.M. Dudley, Nature Physics 6, 790-795 (2010).

[11] K. Hammani, B. Kibler, C. Finot, P. Morin, J. Fatome, J.M. Dudley, and G. Millot, Opt. Lett. 36, 112-114 (2011).

[12] M. Onorato, S. Residori, U. Bortolozzo, A. Montina, and F.T. Arecchi, Phys. Rep. 528, 47-90 (2013).

[13] A. Bendahmane, A. Mussot, P. Szriftgiser, O. Zerkak, G. Genty, J. M. Dudley, and A. Kudlinski, Opt. Lett. 39, 4490-4493 (2014).

[14] J. M. Dudley, F. Dias, M. Erkintalo, and G. Genty, Nat. Phot. 8, 755-764 (2014).

[15] N. Akhmediev, B. Kibler, F. Baronio, M. Belic, W.P. Zhong, Y. Zhang, W. Chang, J. M. Soto-Crespo, P.
Vouzas, P. Grelu, C. Lecaplain, K. Hammani, S. Rica, A. Picozzi, M. Tlidi, K. Panajotov, A. Mussot, A. Bendahmane, P. Szriftgiser, G. Genty, J. Dudley, A. Kudlinski, A. Demircan, U. Morgner, S. Amiraranashvili, C. Bree, G. Steinmeyer, C. Masoller, N. G. R. Broderick, A. F. J. Runge, M. Erkintalo, S. Residori, U. Bortolozzo, F. T. Arecchi, S. Wabnitz, C. G. Tiofack, S. Coulibaly, and M. Taki, J. Opt. 18, 063001 (2016).

[16] R.W. Boyd, Nonlinear Optics (Elesevier, 2008).

[17] C.R. Menuyk, R. Schiek, and L. Torner, J. Opt. Soc. Am. B 11, 2434-2443 (1994).

[18] S. Trillo and P. Ferro, Opt. Lett. 20, 438-440 (1995).

[19] R. Schiek, Y. Baek, and G. I. Stegeman, Phys. Rev. E 53, 1138-1141 (1996).

[20] F. Baronio, C. De Angelis, P.H. Pioger, V. Couderc, and A. Barthelemy, Opt. Lett. 29, 986-988 (2004).

[21] P. Di Trapani, D. Caironi, G. Valiulis, A. Dubietis, R. Danielius, and A. Piskarskas, Phys. Rev. Lett. 81, 570573 (1998).

[22] J. Moses and F. W. Wise, Phys. Rev. Lett. 97, 073903 (2006).

[23] F. Baronio, C. De Angelis, M. Marangoni, C. Manzoni, R. Ramponi, and G. Cerullo, Opt. Express 14, 4775-4779 (2006).

[24] M. Bache, O. Bang, J. Moses, and F. W. Wise Opt. Lett. 32, 2490-2492 (2007).

[25] X. Zeng, H. Guo, B. Zhou, and M. Bache, Opt. Express. 20, 27071-27082 (2012).

[26] M. Conforti, F. Baronio, and S. Trillo, Opt. Lett. 37, 1082-1084 (2012).

[27] R. A. Fuerst, D.M. Baboiu, B. Lawrence, W. E. Torruellas, G. I. Stegeman, S. Trillo, and S. Wabnitz, Phys. Rev. Lett. 78, 2756-2759 (1997).

[28] H. Fang, R. Malendevich, R. Schiek, and G. I. Stegeman, Opt. Lett. 25, 1786-1788 (2000). 